\title{
Análisis del desempeño cognitivo de los enfermos con Parkinson: Importancia del nivel educativo.
}

\author{
Analysis of cognitive performance in patients with Parkinson disease: The importance of \\ educational level.
}

\author{
Lilia Mestas Hernández ${ }^{\text {a }}$, Judith Salvador Cruz ${ }^{\text {b }}$ \\ ${ }^{a}$ Facultad de Estudios Superiores Zaragoza, Universidad Nacional Autónoma de México. México DF. ${ }^{b} F a$ - \\ cultad de Estudios Superiores Zaragoza, Universidad Nacional Autónoma de México. México DF.
}

Correspondencia:Lilia Mestas (lilia_mestas@yahoo.com.mx)

Recibido: 18/07/2012; aceptado con modificaciones: 07/01/2013

RESUMEN: Introducción. Las experiencias vitales, entre ellas la educación, capacitan a las personas para tolerar los efectos de una patología. En la enfermedad de Parkinson (EP) esta reserva cognitiva podría estar beneficiando el rendimiento cognitivo de los pacientes y actuando con un atenuador de los síntomas derivados de una posible demencia asociada. El objetivo de este trabajo es el de estudiar el efecto del nivel de educación sobre el rendimiento cognitivo en una muestra de pacientes con EP, e investigar si existe relación entre el nivel de educación adquirido, el deterioro cognitivo y el inicio de la enfermedad.

Pacientes y método: La muestra estuvo compuesta por 30 pacientes con diagnóstico de Parkinson sin demencia, que fueron distribuidos en tres grupos dependiendo de su nivel de escolaridad (básica, media y superior), y a los que se les aplicó el MiniMental Parkinson (MMP) como medida de rendimiento cognitivo, y la escala autoaplicada para la evaluación de la depresión con el Inventario de Depresión de Beck (BDI), con el fin de descartar trastornos depresivos.

Resultados: Se obtuvieron diferencias significativas entre el nivel de educación básico y los niveles medio y superior ( $p=0,016, p=0,003$, respectivamente). No se encontraron efectos significativos con la edad de inicio de la EP entre los tres grupos analizados. El análisis de las subpruebas del MMP no arrojó diferencias significativas; sin embargo, se obtuvo una correlación moderada entre la subprueba de memoria y los años de escolaridad $(r=0,407, p<0,005)$.

Conclusiones: El nivel de educación puede estar afectando al rendimiento cognitivo en la vejez y funcionar como un atenuador de los síntomas, mejorando la habilidad adquirida del cerebro a través de la experiencia (e.g., educación), para tolerar los efectos de una patología.

PALABRAS CLAVE: Deterioro cognitivo, Enfermedad de Parkinson, Nivel de educación, Reserva cognitiva.
ABSTRACT: Introduction: Vital experiences, among them education, enable people to tolerate the effects of pathology. In Parkinson disease (PD) this cognitive reserve could benefit the patient's cognitive performance and could act as an attenuator of derived symptoms from a possible associated dementia. The purpose of this study is to determine the effect of educational level on the cognitive performance in a sample of patients with PD and to investigate if there is any type of relation between acquired educational level, cognitive deterioration and the beginning of the disease.

Patients and method: the sample consisted of 30 patients diagnosed with Parkinson disease without dementia, that were distributed in three groups according to their educational level (basic, middle and high), they were assessed with the Mini Mental Parkinson (MMP) test as a measurement method for the cognitive performance, and the self applied Beck's Depression Inventory (BDI) scale to discard depressive disorders.

Results: We obtained significant differences between the basic, the middle and the superior educational levels ( $p=0,016, p=0,003$, respectively). We didn't find significant effects differences involving the age of beginning of PD among the three groups analyzed. The analysis made to the subtests of the MMP didn't show significant differences either. However, we obtained a moderate correlation between the memory subtest and the educational levels $(r=0,407, p<0,005)$.

Conclusions: The academic level may be affecting at some degree the cognitive performance in old age and act as an attenuator for the neurological pathology symptoms, improving the acquired brain abilities through experience (e.g. education). These results are interpreted in favor for the cognitive reserve hypothesis.

KEY WORDS: Cognitive impairment, Parkinson Disease, Educational status, Cognitive reserve. 


\section{Introducción}

La enfermedad de Parkinson (EP) es un trastorno neurodegenerativo que se caracteriza por un desorden en los movimientos, con una relativa preservación de las habilidades intelectuales (1). No todas las personas que tienen Parkinson llegan a sufrir demencia, y cuando ocurre se produce de manera habitual tras años de evolución o en inicios tardíos de la enfermedad. Sin embargo, una vez que inicia, existe una probabilidad seis veces mayor de padecer demencia respecto a las personas sanas (2). En términos más generales, y según diferentes estudios, la demencia aparece en un rango que oscila entre el 10 y $95 \%$ de los enfermos con Parkinson, dependiendo de la población estudiada, del criterio y método de diagnóstico utilizado (3-5). Se diferencia de la demencia con cuerpos de Lewy por la mayor gravedad y frecuencia de ésta en la disfunción ejecutiva y los síntomas psiquiátricos $(6,7)$. Esta distinción es necesaria para el diagnóstico diferencial dado que en ambas se observa la presencia de este tipo de inclusiones citoplasmáticas -cuerpos de Lewy- en las neuronas corticales.

Recientes estudios consideran que el deterioro cognitivo leve (DCL) observado en los pacientes con Parkinson, puede referirse a otra condición clínica que estaría en un lugar intermedio entre la funcionalidad cognitiva normal y la demencia (8). Las diferencias en los déficits cognitivos entre los pacientes de Parkinson con DCL y demencia han sido consideradas de carácter cuantitativo, hecho refrendado por las diferencias entre estos grupos mediante análisis de la actividad electroencefalográfica (9). El hecho de que los déficits cognitivos encontrados en pacientes con y sin demencia en la EP sean cualitativamente iguales induce a pensar en la posibilidad de que los factores sociodemográficos estén actuando como mediadores, sino del inicio, sí del nivel de deterioro cognitivo observado en este tipo de pacientes. Entre estos factores, el nivel de educación ha sido tratado desde diferentes perspectivas experimentales que conciben la educación como una actividad ejercitadora de la funcionalidad cognitiva (10), que podría atenuar la gravedad de los déficits cognitivos en diferentes trastornos neurológicos. En este sentido la hipótesis de la reserva cognitiva (RC) (11-14), plantea la posibilidad de que la habilidad del cerebro para tolerar mejor los efectos de una patología asociada a la demencia, sea el resultado de una habilidad innata o de los efectos de las experiencias vividas, como la educación o la ocupación laboral (15).

La educación favorece el bienestar de las personas desde diferentes vertientes. Los niveles de educación altos se han relacionado con tasas de mortalidad más bajas, mejor salud y un mejor desempeño cognitivo durante la vejez, respecto a las personas con niveles de educación bajos (16). Un hecho importante y relacionado es que el incremento en los niveles de educación viene asociado a una mayor tasa de empleo (17), a un mayor poder adquisitivo $(18,19)$, y de igual manera influye 
ORIGINALES Y REVISIONES

sobre el cuidado de la salud, el medio ambiente, la exposición y el estilo de vida $(20,21)$. Todos estos factores determinan que en la vejez se produzcan diferencias estructurales y en la actividad cerebral (22), que podrían propiciar un mejor rendimiento cognitivo y por lo tanto actuar como factores atenuadores del declive inherente a la vejez.

Un reciente estudio longitudinal, realizado por EclipSe Collaborative Members (23), informa que a mayor nivel de educación se asocia una menor probabilidad de tener demencia en un futuro. Pero al mismo tiempo, el nivel de educación no se ha relacionado con la probabilidad de desarrollar algún tipo de patología neurodegenerativa. En otros estudios se ha constatado que el género femenino, la edad superior a 65 años, el menor nivel de educación y el número de hijos son las variables sociodemográficas que más influyen en el estado cognitivo (24). La hipótesis del deterioro cognitivo con la edad se deriva de cambios fisiológicos en la estructura y funcionalidad del lóbulo frontal (25), tal como se ha comentado en párrafos anteriores, y a tenor de las últimas investigaciones realizadas, estos cambios podrían ser modulados por el nivel de educación del sujeto (26) y la complejidad de las tareas realizadas durante su vida (27).

A la luz de estos datos y respecto a la EP, se infiere que el nivel de educación podría no tener relación con la probabilidad de padecer la enfermedad, pero sí con el rendimiento cognitivo y al desarrollo de una demencia asociada. Entendiendo la educación como una actividad que permite incrementar la reserva cognitiva, planteamos la hipótesis de que habría diferencias significativas en el rendimiento cognitivo en los diferentes niveles de educación, lo que estaría indicando la influencia de la educación sobre el desempeño cognitivo de los pacientes. Por otro lado, se espera que la variable Edad de inicio, no muestre diferencias significativas entre los grupos, evidenciado así que el nivel de educación no estaría afectando al momento vital de inicio de la EP, y por lo tanto no tendría un efecto protector sobre dicha patología pero sí, y de cumplirse la primera hipótesis, sobre el deterioro cognitivo de la EP y su posible evolución a demencia.

\section{Método}

\section{Participantes}

Se incluyeron 30 pacientes con diagnóstico de EP, y con un deterioro motor entre el estadio I y II, medido a través de la escala de Hoehn y Yahr (28). Tras dar su consentimiento informado se distribuyeron en tres grupos dependiendo de los niveles de educación (básica, media, superior). Los datos sociodemográficos de los participantes se muestran en la tabla 1. 


\section{Instrumentos}

Se utilizó el MiniMental Parkinson (MMP) (29), que es una prueba de análisis breve y estandarizada, que permite comprobar el estado mental de los sujetos con Parkinson. Esta prueba se constituye en siete subsecciones con una puntuación máxima de 32. Para el análisis de las subpruebas se agruparon de la siguiente forma (30).

Orientación (orientación temporal y espacial). Constituida por 10 ítem, cinco de orientación temporal y 5 espacial. A cada respuesta correcta se le da un punto.

Memoria (memoria visual inmediata y de evocación). El recuerdo visual consiste en mostrar al paciente tres tarjetas con sólo una de las dos figuras que se presentaron inicialmente. Éstas se presentan simultáneamente y se le pide que recuerde la figura y el orden inicial en el que se presentaron. Se asigna un punto por cada figura que recuerde correctamente y un punto extra por evocar el orden correcto de las tarjetas.

Atención (atención y control mental). La atención y el control mental se exploran de acuerdo al diseño clásico de Folstein (31), es decir, una serie de restas de 100 menos 7 y retrocediendo cinco veces; se otorga un punto por cada resta correcta.

Fluencia verbal. Se solicita al paciente el nombre de tres animales que comience con la misma letra en un tiempo máximo de 30 segundos. Se obtiene un punto por cada palabra correcta.

Abstracción (procesamiento de conceptos y flexibilidad cognitiva). El cambio de set (escenario) o flexibilidad cognitiva consiste en presentar una carta con cuatro figuras y preguntar en qué se diferencia cada figura de las otras tres; el sujeto debe responder a las diferencias de color, tamaño, orientación y forma; se asigna un punto por cada diferencia declarada. El procesamiento de conceptos consiste en tres grupos de tres palabras. En cada grupo, dos palabras pertenecen a una categoría semántica (frutas, ropa, medios de transporte) y la tercera no está relacionada. Se debe indicar cuál de las dos palabras del triplete están más relacionadas. Se asigna un punto por cada asociación correcta.

También se incluyó la escala autoaplicada para la evaluación de la depresión (Inventario de Depresión de Beck; IDB) (32), con el fin de excluir aquellos sujetos que manifestaran un trastorno depresivo. Es un instrumento de 21 preguntas con respuesta precodificada para evaluar la intensidad de los cuadros depresivos. Las puntuaciones que corresponden a las categorías del IDB son las siguientes: 1) menos de 10 puntos, depresión nula o mínima, 2) entre 10 y 18 puntos -depresión leve o moderada-, 3) entre 19 y 29 puntos -depresión moderada a grave-, 4) $30 \mathrm{o}$ más puntos -depresión grave-. Se obtuvo una media de 6,93 $(D T=3,45)$ en los 30 participantes. Ninguno de ellos supero la puntuación de 10. 
Tabla 1.

Características demográficas de la muestra.

\begin{tabular}{|l|c|}
\hline \multirow{2}{*}{ Casos } & Enfermedad de Parkinson \\
\cline { 2 - 2 } Edad (años) & 30 \\
\hline Sexo(masculino/femenino) & $\mathrm{M}=64,53(\mathrm{DT}=6,14)$ \\
\hline Escolaridad (años) & $\mathrm{M}=9,47(\mathrm{DT}=5,26)$ \\
\hline Enseñanza básica (3-6 años) & $8(26,66 \%)$ \\
\hline Enseñanza media (6-9 años) & $11(36,67 \%)$ \\
\hline Enseñanza superior (9 años ó más) & $11(36,67 \%)$ \\
\hline Evolución enfermedad (años) & $\mathrm{M}=9(\mathrm{DT}=5,64)$ \\
\hline Edad de inicio & $\mathrm{M}=55,53(\mathrm{DT}=7,38)$ \\
\hline
\end{tabular}

\section{Análisis estadísticos}

Análisis general. Se realizaron ANOVAS de un factor para comprobar los efectos de la variable nivel de educación (bajo, medio, alto) sobre las puntuaciones obtenidas en el MMP, el cuestionario de depresión de Beck y la edad. El nivel de educación se registró a partir de los años de escolaridad (tabla 1). Los análisis sobre las puntuaciones obtenidas en el BDI y la edad se realizaron para confirmar que no había diferencias entre los grupos que pudieran afectar a la interpretación de los resultados.

Análisis específico. Se realizaron ANOVAS de un factor para comprobar los efectos de la variable nivel de educación (bajo, medio, alto) sobre las puntuaciones obtenidas en los agrupamientos de las subpruebas del MMP (Orientación, Memoria, Atención, Fluencia verbal, Abstracción). Por último se realizó un análisis de correlaciones entre el nivel de educación y las subpruebas del MMP.

\section{Resultados}

Se obtuvieron diferencias significativas en el MMP para los diferentes niveles de educación. La comparación por pares informa que las diferencias se dan entre el nivel de educación bajo y los niveles medio y alto (Bonferroni, $p=0,016, p=$ 0,003 , respectivamente). Un análisis más específico sobre las subpruebas del MMP no arrojó diferencias significativas entre las medias de los grupos en los diferentes niveles de educación (tabla 2). Por último, se obtuvieron diferencias significativas 
en las correlaciones bivariadas realizadas sólo entre el nivel de educación y la subprueba del MMP relativa a la memoria [Orientación $(r=-0,153, p>0,05)$; memoria $(r=0,407, p<0,05)$; atención $(r=0,196, p>0,05)$; fluencia $(r=0,035, p>0,05)$; abstracción $(r=0,197, p>0,05)]$.

Tabla 2.

Valores medios obtenidos en las variables analizadas en relación a los niveles de escolaridad (Básica, Media y Superior), y ANOVA univariante.

\begin{tabular}{|c|c|c|c|c|c|c|c|}
\hline Edad & $\begin{array}{c}\text { Básica } \\
66,63 \\
(6,28)\end{array}$ & $\begin{array}{l}\text { Media } \\
63,55 \\
(6,64)\end{array}$ & $\begin{array}{c}\text { Superior } \\
64,00 \\
(5,73)\end{array}$ & $\begin{array}{c}F \\
0,63\end{array}$ & $\begin{array}{c}p \\
0,539\end{array}$ & $\begin{array}{c}r^{2} \\
0,05\end{array}$ & $\begin{array}{c}P \\
0,15\end{array}$ \\
\hline BDI & $\begin{array}{c}7,75 \\
(4,40)\end{array}$ & $\begin{array}{c}7,00 \\
(2,37)\end{array}$ & $\begin{array}{c}6,27 \\
(3,80)\end{array}$ & 0,42 & 0,668 & 0,03 & 0,11 \\
\hline Inicio & $\begin{array}{l}59,50 \\
(6,00)\end{array}$ & $\begin{array}{l}54,82 \\
(6,05)\end{array}$ & $\begin{array}{l}53,36 \\
(8,82)\end{array}$ & 0,63 & 0,189 & 0,12 & 0,34 \\
\hline MMP & $\begin{array}{l}23,75 \\
(3,01)\end{array}$ & $\begin{array}{l}27,73 \\
(3,10)\end{array}$ & $\begin{array}{l}28,55 \\
(2,38)\end{array}$ & 7,27 & 0,003 & 0,35 & 0,91 \\
\hline Orientación & $\begin{array}{c}9,88 \\
(0,35)\end{array}$ & $\begin{array}{c}9,82 \\
(0,41)\end{array}$ & $\begin{array}{c}9,64 \\
(0,67)\end{array}$ & 0,59 & 0,560 & 0,04 & 0,14 \\
\hline Memoria & $\begin{array}{r}4,25 \\
(1,67)\end{array}$ & $\begin{array}{c}5,00 \\
(1,41) \\
\end{array}$ & $\begin{array}{c}5,73 \\
(1,55) \\
\end{array}$ & 2,16 & 0,135 & 0,14 & 0,40 \\
\hline Atención & $\begin{array}{r}2,38 \\
(1,77)\end{array}$ & $\begin{array}{c}3,82 \\
(1,54)\end{array}$ & $\begin{array}{c}3,91 \\
(1,45)\end{array}$ & 2,65 & 0,089 & 0,16 & 0,48 \\
\hline Fluencia & $\begin{array}{c}2,50 \\
(0,76) \\
\end{array}$ & $\begin{array}{c}2,82 \\
(0,40)\end{array}$ & $\begin{array}{c}2,73 \\
(0,65)\end{array}$ & 0,66 & 0,524 & 0,05 & 0,15 \\
\hline Abstracción & $\begin{array}{c}4,75 \\
(3,10)\end{array}$ & $\begin{array}{c}6,27 \\
(1,01)\end{array}$ & $\begin{array}{c}6,55 \\
(1,04)\end{array}$ & 2,53 & 0,099 & 0,16 & 0,46 \\
\hline
\end{tabular}

MMP: minimental Parkinson

BDI: inventario de depresión de Beck

* La desviación típica se pone entre paréntesis 
Los resultados obtenidos informan de una relación entre el número de años de escolaridad y la puntuación total obtenida en el MMP, cuya correlación ( $\mathrm{r}=$ $0,399, \mathrm{p}<0,05)$ se evidencia gráficamente en la figura 1.

Figura 1.

Relación entre la puntuación obtenida en el Minimental Parkinson (MMP) y los años de escolaridad.

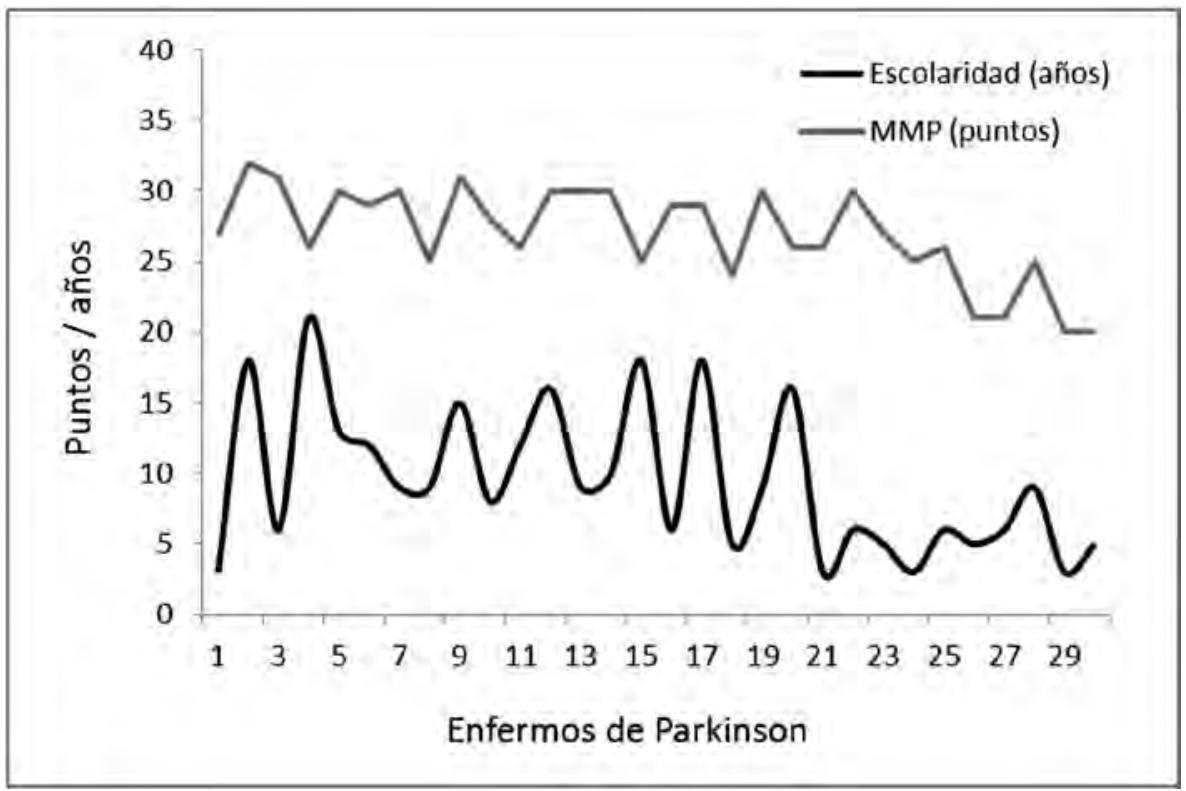

Discusión y conclusiones

Los resultados obtenidos señalan que el deterioro cognitivo y su posible evolución a demencia en la EP, podría esta relacionado positivamente con el nivel de educación de los participantes, mientras que no se obtuvieron resultados que evidencien una relación entre el nivel de educación y la edad de inicio de la EP. Por otro lado, el análisis de las subpruebas no arrojó resultados significativos, seguramente debido al pequeño número de la muestra y a la escasa variabilidad de las puntuaciones; sin embargo, previendo esta contingencia se realizó un análisis de correlaciones que, si bien no permite extraer relaciones causales sí nos informan, por su menor exigencia, de una correlación significativa entre la subprueba de memoria y los años de escolaridad.

Estos resultados son congruentes con la hipótesis de la reserva cognitiva (1114), como un mecanismo que asocia los bajos niveles educativos con un mayor 
riesgo de padecer un proceso neurodegenerativo. La capacidad del cerebro, o reserva cognitiva, para tolerar los efectos de una patología, puede ser el resultado de una habilidad innata o el efecto de las experiencias vividas, como la educación o la ocupación laboral (15). La educación resulta una actividad relevante y representativa del desarrollo cerebral. El aprendizaje de la lectura y la escritura comportan cambios en la arquitectura funcional del cerebro, lo que permitiría compensaciones activas frente a los cambios relacionados con el envejecimiento (33). De igual manera, la alfabetización ayuda a aumentar las conexiones nerviosas junto a otros factores que estarían determinando un "ambiente enriquecido" (34). Sin embargo, la alfabetización puede provenir de otras fuentes diferentes a la experiencia educativa; es decir, se pueden tener más oportunidades durante la vida para mejorar la alfabetización que no tendría su reflejo en los años de educación. Manly et al. (33), concluyen que los años de educación pueden estar representando la reserva cognitiva siempre que se estimen como fruto de la experiencia.

La reserva cognitiva pretende explicar la falta de correspondencia observada entre la cantidad de daño o patología cerebral y sus manifestaciones clínicas (35). Una cuestión importante que se deduce de esta aproximación al problema es la necesidad de conseguir un método de medida de la reserva cognitiva fiable y que habilite el estudio de métodos para incrementarla o preservarla, al tiempo que permita mayor precisión a los sistemas de diagnóstico. Esto tiene sentido ya que la reserva cognitiva tiene una alta variabilidad entre las personas que, precisamente, puede normalizarse siempre y cuando conozcamos y registremos, a través de instrumentos adecuados, qué factores son los que intervienen en su unidad como constructo a estudiar. Hasta la fecha se ha recurrido a diferentes estimaciones para su medida, referidas bien sólo a la inteligencia premórbida $(36,37)$, a la combinación de ésta, con los años de educación y el nivel ocupacional (38-40), o a la inteligencia premórbida y los años de educación (40).

Para concluir y tal como opina Stern (41), las experiencias de la vida, entre ellas la educación, producen modificaciones anatómicas a nivel de las redes neuronales. Se constituye así como un proceso dinámico que evoluciona con la edad y que tiene implicaciones cruciales al momento de enfrentar una disfunción cerebral. Los resultados encontrados en esta investigación son congruentes con este supuesto en tanto se observan diferencias significativas por nivel de educación en las puntuaciones obtenidas en el MMP en una muestra de enfermos con Parkinson. Las correlaciones realizadas entre los años de escolaridad y las subpruebas del MMP, muestran una correlación significativa tan sólo con la memoria. En suma, y a partir de estos resultados, se observa que la educación proporcionaría un incremento en la reserva cognitiva que daría lugar a un mejor rendimiento cognitivo en la edad adulta, hecho que resultaría más relevante si se presentara una enfermedad neurológica como el Parkinson, y donde la memoria, como en muchos otros trastornos 
ORIGINALES Y REVISIONES

(42), podría resultar un indicador eficaz no sólo como síntoma del deterioro, sino también como marcador de reserva cognitiva.

Los resultados recabados en este trabajo deben tomarse con cautela dado el pequeño número de pacientes analizado, además, la variabilidad de los valores obtenidos en el MMP no indica necesariamente que los sujetos que puntuaron más bajo vayan a desarrollar demencia en los próximos años. A pesar del pequeño número de pacientes la utilización de una prueba del deterioro cognitivo específica para esta patología, como el MMP, proporciona una alta fiabilidad y consistencia con los resultados obtenidos. Si bien, como apuntan Villarejo y Puertas-Martín (43), no existe el test ideal, su utilización debe depender de la disponibilidad de tiempo y la experiencia del experimentador. En este caso se requería de una prueba rápida, fiable y validada, como así ha sido, por personal altamente preparado y con experiencia en su aplicación. Futuras investigaciones de corte longitudinal, deberían estudiar en qué sentido afecta el nivel de educación en la evolución de los déficit cognitivos hacia una demencia en los enfermos con Parkinson, tal como de manera más general se ha planteado en el estudio de EclipSe Collaborative Members (23); con la finalidad de confirmar los resultados mostrados en este trabajo y un posible efecto protector de los niveles de educación sobre el desarrollo de una demencia asociada al Parkinson.

En definitiva, este trabajo abunda en las conclusiones de Poletti, Emre y Bonuccelli (44), donde se advierte de la necesidad de comprender cómo la reserva cognitiva, a través del nivel de escolaridad, se convierte en un factor modulador del deterioro cognitivo presente en la EP. Por otro lado, los resultados obtenidos tras aplicar el MMP, son congruentes con recientes investigaciones que han utilizado el Mini-Mental State Examination (45), prueba de la que deriva el MMP y que por lo tanto, se convierte en una clara referencia de comparación (46).

BIBLIOGRAFÍA:

(1) Almeida GM, Correa L, Letro GH, Souza A, Bastos A. Dementia and mild cognitive impairment in patiens with Parkinson's Disease. Arq Neuropsiquiatr 2009; 67(2-B): 423-7.

(2) Aarsland D, Andersen K, Larsen JP, Lolk A, Nielsen H, Kragh-Sorensen P. Risk of dementia in Parkinson's disease: a community-based, prospective study. Neurology 2001; 56: 730-6.

(3) Aarsland D, Andersen K, Larsen JP, Lolk A, Kragh-Soremsen P. Prevalence and characteristics of dementia in Parkinson's disease: an 8-year prospective study. Arch Neurol 2003; 60: 387-92.

(4) Emre M. Dementia associated with Parkinson's disease. Lancet Neurol 2003; 2: 229-37.

(5) Verbaan D, Marinus J, Visser M, van Rooden SM, Stiggelbout AM, Middelkoop HA, et al. Cognitive impairment in Parkinson' disease. J Neurol Neurosurg Psychiatry 2007; 78: 1182-7. 
(6) Aarsland D, Ballard CG, Halliday G. Are Parkinson's Disease with dementia and Dementia with lewy Bodies the Same Entity? J Geriatr Psychiatry Neurol 2004; 17: 137-45.

(7) Diéguez E, Charro C, Hortigüela RR. A la sombra de la enfermedad de Alzheimer crecen brotes de enfermedad de Parkinson y demencia por cuerpos de Lewy. Psicogeriatría 2010; 2(4): 221-6.

(8) Janvin CC, Larsen JP, Aarsland D, Hugdahl K. Subtypes of mild cognitive impairment in Parkinson's disease: progression to dementia. Mov Disord 2006; 9: 1343-9.

(9) Fonseca LC, Tedrus GM, Letro GH, Bosson AS. Dementia, mild cognitive impairment and quantitative EEG in patients with Parkinson's disease. Clin EEG Neurosci 2009; 40(3): 168-72.

(10) Luria, A. R. (1987). El desarrollo histórico de los procesos cognitivos. Tres Cantos: Akal

(11) Stern Y. Cognitive reserve and Alzheimer disease. Alzheimer Dis Assoc Disord 2006; 20 : S69-74.

(12) Valenzuela MJ, Sachdev P. Brain reserve and cognitive decline: a non-parametric systematic review. Psychol Med 2006; 36: 1065-73.

(13) Valenzuela MJ, Breakspear M, Sachdev P. Complex mental activity and the aging brain: molecular, cellular and cortical network mechanisms. Brain Res Rev 2007; 56: 198-213.

(14) Valenzuela MJ, Sachdev P, Wen W, Chen X, Brodaty H. Lifespan mental activity predicts diminished rate of hippocampal atrophy. PLoS One. 2008;3:e2598.

(15) Rodríguez MR, Sánchez, JL. (2004). Reserva cognitiva y demencia. Anales de Psicología 2004; 20(2): 175-86.

(16) Kinsella K, He W. An Aging World: 2008. International Population Reports. 2009.

(17) Asghar Z, Fuchs M. Transition From Work to Retirement in EU25. Policy Brief December 2006. Vienna: European Centre for Social Welfare Policy and Research. 2006.

(18) Chawla M, Betcherman G, Banerji A, Bakilana AM, Feher C, Mertaugh M, et al. From red to gray: Third transition of aging populations in eastem Europe and the former Soviet Union, The World Bank. 2007.

(19) Day JC, Newburger EC. The Big Payoff: Educational Attainment and Synthetic Estimates of Work-Life Earnings. U.S. Census Bureau Current Population Report P23-210. Washington, DC: U.S. Government Printing Office. 2002.

(20) Adler NE, Newman K. 2002. Socioeconomic Disparities in Health: Pathways and Policies. Health Affairs 2002; 21(2): 60-76.

(21) Mirowsky J, Ross CE. Education, Personal Control, Lifestyle and Health: A Human Capital Hypothesis." Research on Aging 1988; 20(4): 415-49.

(22) Ho AJ, Raji CA, Beker JT, López OL, Kuller LH., Hua X, et al. The effects of physical activity, education, and body mass index on the aging brain. Human Brain Mapping 2010; 32(9): 13711382.

(23) EclipSe Collaborative Members. Education, the brain and dementia: neuroprotection or compensation? Brain 2010; 133: 2210-16.

(24) Mías CD, Sassi M, Masih ME, Querejeta A, Krwchik R. Deterioro cognitivo leve: estudio de prevalencia y factores sociodemográficos en la ciudad de Cordoba, Argentina. Rev Neurol 2007; 44(12): $733-8$.

(25) Woodruff-Pak DS. The Neuropsychology of aging. Malden: Blackwell Publishers: 1997.

(26) Fichman HC, Fernandes CS, Nitrini R, Lourenco RA, de Paiva EM, Cathery-Goulart MT, et al. Age and educational level effects on the performance of normal elderly on category verbal fluency tasks. Dement. Neuropsychol 2009; 3(1): 49-54. 
ORIGINALES Y REVISIONES

(27) Finkel D, Andel R, Gatz M, Pedersen NL. The Role of Occupational Complexity in Trajectories of Cognitive Aging Before and After Retirement. Psychol Aging 2009; 24(3): 563-73.

(28) Hoehn M, Yahr M. Parkinsonism: onset, progression and mortality. Neurology 1967; 17: 427-442.

(29) Mahieux F, Michelet D, Manifacier M, Boller F, Fermanian J, Guillard A. Mini-mental Parkinson: first validation study of a bedside test constructed for Parkinson's disease. Behav Neurol 1995; 8: $15-22$.

(30) Parrao-Díaz T, Chaná-Cuevas P, Juri-Claverías C, Kunstmann C, Tapia-Núñez J. Evaluación del deterioro cognitivo en una población de pacientes con enfermedad de Parkinson mediante el Test minimental Parkinson. Rev Neurol. 2005; 40 (6):339-44.

(31) Folstein MF, Folstein SE, McHugh PR. "Mini-Mental State": A practical method for grading the cognitive state of patients for the clinician. J. Psychiatr. Res. 1975; 12:397-408.

(32) Beck AT, Ward CH, Mendelson M, Mock J, Erbaugh J. An inventory for measuring depression. Arch Gen Psychiatry. 1961;4:53-63.

(33) Manly J, Touradji P, Tan M-X, Stern Y. Literacy and Memory Decline Among Etnnically Diverse Elders. J Clin Exp Neuropsychol. 2003;25:680-90.

(34) Diamond MC. Enriching heredity: The impact of theenvironment om the anatomy of the brain. New York: The Free Press. 1988.

(35) Stern Y. What is cognitive reserve? Theory and research application of the reserve concept. J Int Neuropsychol Soc 2002; 8: 448-60.

(36) Corral M, Rodriguez M, Amenedo E, Sanchez JL, Diaz F. Cognitive reserve, age, and neuropsychological performance in healthy participants. Dev Neuropsychol 2006; 29: 479-91.

(37) León I, García J, Tapia L. Construcción de una escala de reserva cognitva en población española: estudio piloto. Rev Neurol 2011; 52(11): 653-60.

(38) Pereda M, Ayuso-Mateos JL, Gomez A, Echevarria S, Farinas MC, García D, et al. Factors associated with neuropsychological performance in HIV-seropositive subjects without AIDS. Neuropsychol Med 2000; 30: 205-17.

(39) Sánchez JL, Rodríguez M, Carro J. Influence of cognitive reserve on neuropsychologic functioning in Alzheimer's disease type sporadic in subjects of Spanish nationality. Neuropsychiatry Neuropsychol Behav Neurol 2002; 15: 113-22.

(40) Santamarina P, Corral M. Influencia de la reserva cognitiva en el rendimiento neuropsicológico de los pacientes con epilepsia. Med Clin 2009; 132: 459-62.

(41) Stern Y. Cognitive Reserve. Neuropsychologia 2009; 47: 2015-28.

(42) Mestas L, Salvador J, Gordillo F. Reserva cognitiva y déficit en la planificación en pacientes con esclerosis múltiple. Rev. Asoc. Esp. Neuropsiq 2012; 32(113): 55-65.

(43) Villarejo A, Puertas-Martín V. Utilidad de los test breves en el cribado de demencia. Neurología 2010; 26(7): 425-33.

(44) Poletti M, Emre M, Bonuccelli U. Mild cognitive impairment and cognitive reserve in Parkinson's disease. Parkinsonism Relat Disord. 2011; 17(8): 579-86.

(45) Kierzynka A, Kaźmierski R, Kozubski W. Educational level and cognitive impairment in patients with Parkinson disease. Neurol Neurochir Pol 2011; 45(1): 24-31.

(46) Larner AJ. Mini-mental Parkinson (MMP) as a dementia screening test: comparison with the Mini-Mental State Examination (MMSE). Curr Aging Sci 2012; 5(2): 136-9. 